\title{
Successful pregnancy outcomes following in-vitro fertilization in infertile couples with high levels of antisperm antibodies
}

\author{
Varuni Tennakoon ${ }^{1}$, Deepal S Weerasekera ${ }^{1}$, Surangi G Yasawardene ${ }^{2}$ \\ Sri Lanka Journal of Obstetrics and Gynaecology 2010; 32: 47-48
}

\section{Introduction}

The role of antisperm-antibodies (ASA) as a cause of human infertility was recognized with evidence provided by research through last few decades. It is established that ASA can negatively impact fertility by affecting sperm motility, cervical mucus penetration, gamete fusion and potentially even the first steps of embryo development ${ }^{1}$. ASA can be found in systemically (in blood and lymph) and in local secretions (in semen, oviductal, uterine, cervicalvaginal and follicular fluid) $)^{2,3}$. The three major structural types of antisperm immunoglobulins are IgA, IgG and $\operatorname{IgM}^{2}$. With the development and the encouraged use of accurate and easy assays for the screening of ASA and the progress in assisted reproductive technologies (ART), the role of ASA in infertility as well as the potential for their treatment is becoming better defined ${ }^{3}$. It is believed that the degree of sperm-autoimmunization is the main empirical criterion in choosing more or less invasive ART procedures for couples with $\mathrm{ASA}^{3}$. In-vitro fertilization (IVF) with conventional IVF/intra-cytoplasmic sperm injection (ICSI), bypasses most, if not all, of the steps in conception that are adversely affected by ASA in human infertility ${ }^{1,4}$. We report three cases of infertile couples who demonstrated a high level of antisperm antibodies, and were successful in achieving pregnancies following in-vitro fertilization (IVF). Apart from infertility, those couples were otherwise healthy and free of any significant illnesses. The ASA were detected using mixed agglutination reaction latex bead test (SpermMAR, Fertipro NV, Belgium).

\section{Case 1}

A 39-year old lady married for seven years presented with primary subfertility of same duration and had undergone several attempts of timed

\footnotetext{
${ }^{1}$ Prarthana Centre for ART, Rajagiriya

${ }^{2}$ Department of Anatomy, Faculty of Medical Sciences, University of Sri Jayawardenepura

Correspondence: Varuni Tennakoon

E-mail:varuni16email@yahoo.com
}

intercourse following follicular tracking and hCG triggering. A diagnostic laparoscopy and dye test had been performed and revealed a normal pelvic anatomy with bilateral patent tubes. Thereafter she had undergone six unsuccessful intrauterine inseminations (IUI). Her husband, a 39-year old, possessed a normozoospermic seminal fluid analysis. When screening for ASA female partner was highly positive ( $>50 \%$ bead binding) for IgA and IgG isotypes of ASA in cervical mucus and serum. Semen was negative for ASA. She was directed to IVF treatment. Five oocytes were recovered and Intra-cytoplasmic sperm injection (ICSI) was performed as the method of fertilization on all oocytes. Her oocyte free follicular fluid was collected and tested for ASA. It also became highly positive for both IgA and IgG isotypes of ASA. One out of five oocytes was fertilized and cleaved. On day 2 of fertilization, embryo transfer was performed. Clinical pregnancy was confirmed at 8 weeks of gestation and she delivered a healthy baby eventually.

\section{Case 2}

A 32-year old lady with her husband (36 years) presented with primary subfertility for six years. She had undergone timed intercourse thrice and six IUIs following a diagnostic laparoscopy and dye test which revealed a normal pelvis with patent tubes. Male partner possessed a normozoospermic seminal fluid analysis. However, he demonstrated a high level ( $>50 \%$ bead binding) of ASA IgA and IgG isotypes on spermatozoa. Female partner was also positive for IgA isotype ( $30 \%$ bead binding) in cervical mucus. Her serum was negative for ASA. The couple underwent IVF, recovering six oocytes. Her follicular fluid was negative for ASA. ICSI was performed on all oocytes. Three embryos were cleaved. Embryo transfer was performed on day 3 of fertilization. Clinical pregnancy was confirmed and she also delivered a healthy baby at term.

\section{Case 3}

A couple, having undergone number of timed inseminations and more than twenty IUIs presented with primary subfertility for 19 years. Female partner, a 40-year old has had two diagnostic laparoscopies 
and dye tests during the course of infertility which revealed again a normal pelvis with bilateral patent tubes. Her husband, a 43-year old, possessed a normozoospermic seminal fluid analysis. Female partner was highly positive ( $>50 \%$ bead binding) for IgG isotype of ASA in serum. Semen was negative for ASA. The couple underwent IVF, recovering four oocytes. Her follicular fluid was highly positive for IgG isotype of ASA. ICSI was performed on two oocytes and conventional IVF was performed on the other two oocytes as the method of fertilization. Three embryos were cleaved. Embryos cleaved from conventional IVF were transferred on day 3 of fertilization. Clinical pregnancy was confirmed and she too delivered a healthy baby at term.

\section{Discussion}

The presence of ASA may impair in vivo sperm fertilizing ability and is an important factor which may prevent the success of various fertility treatments ${ }^{5}$. The incidence of ASA has been estimated to range from $9-36 \%$ in infertile couples depending on population and method of testing, postulates the fact that infertility due to presence of ASA is relatively rare, but not so uncommon ${ }^{1}$. It is also well documented that the presence of high antibody concentrations or titres both locally and systemically suggests a poor prognosis ${ }^{4}$. Therefore it is evident that screening for ASA is critical to avoid repeated unsuccessful treatment procedures, and direct infertile couples to suitable treatment modalities. Fertilization in vitro currently appears to offer an excellent chance to circumvent the negative effects of ASA in cervical mucus, in oviductal, uterine, tubal secretions and in follicular fluid ${ }^{6}$. ICSI on the other hand minimizes the inhibitory effects of ASA on spermatoza on zonapellucida binding and other subsequent events of fertilization ${ }^{5,7,8}$. Numerous studies have shown enhanced fertilization rates and pregnancy rates with IVF/ICSI in couples strongly positive for ASA ${ }^{5,9,10}$. Though these highest level reproductive technologies give the best current prospects for pregnancy in couples with high ASA levels, they also are invasive and costly. It is hoped that further work in the laboratory will give rise to newer, safer, less expensive and effective treatments in the near future.

\section{Conclusion}

It is important to screen infertile couples for the presence of ASA to direct them to suitable treatment modalities. IVF/ICSI is effective in achieving pregnancy in couples possessing high levels of ASA. However, these procedures are invasive and expensive with some risk issues. Further studies on ASA are needed in order for these couples to be treated effectively, efficiently and safely.

\section{References}

1. Lee R, Goldstein M, Ullery BW, Witkin SS, et al. Value of serum antisperm antibodies in diagnosing obstructive azoospermia. Journal of Urology 2009; 181: 264-9.

2. Kutteh WH. Do antisperm antibodies to spermatozoa alter normal reproductive function? Human Reproduction 1999; 14: 2426-9.

3. Francavilla F, Santucci R, Barbonetti A, Francavilla S. Naturally occurring antisperm antibodies in men: Interference with fertility and clinical implications. An update. Frontiers in Bioscience 2007; 12: 2890-911.

4. Chamley LW, Clarke GN. Antisperm antibodies and conception. Semin Immunopathol 2007; 29: 169-84.

5. Lombardo F, Gandini L, Lenzi A, Dondero F. Antisperm immunity in assisted reproduction. Journal of Reprod Immunology 2004; 62: 101-9.

6. Bohring C, Krause W. The role of antisperm antibodies during fertilization and for immunological infertility. Chem Immunol Allergy 2005; 88: 15-26.

7. Marin Briggiler CI. Use of naturally occurring antisperm antibodies for the identification of proteins involved in human gamete interactions. Salud (i) Cinecia 2007; 15: 856-9.

8. Bronson RA. Antisperm antibodies: A critical evaluation and clinical guidelines. Journal of Reprod Immunology 2000; 45: 159-83.

9. Shibahara H, Shiraishi $Y$, Suzuki M. Diagnosis and treatment of immunologically infertile males with antisperm antibodies. Reproductive Medicine and Biology 2005; 4: 133-41. 